\section{Buchrezension zu: Calling Bullshit}

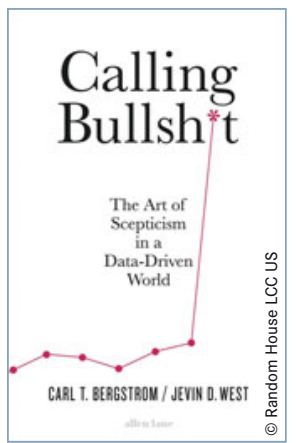

Calling Bullshit

The Art of Skepticism in Data-Driven World

Carl T. Bergstrom und Jevin D. West

318 S., Random House LCC US, 2021. HC, $18,-\$$

ISBN: 9780525509202

Auch als E-Book erhältlich

DOI: $10.1007 / \mathrm{s} 12268-021-1580-\mathrm{x}$

(C) Der Autor 2021

Spätestens seit den vier Jahren der Trump-Präsidentschaft sollte jedem mündigen Staatsbürger (und damit auch jedem Wissenschaftler!) klargeworden sein, dass wir nicht in einem Informationszeitalter leben, sondern in einem Zeitalter der Desinformation, in dem Fakten beliebig für den jeweiligen Zweck zurechtgebogen und über social media verbreitet werden. Es wird zunehmend wichtiger für jeden einzelnen, folgende Fragen zu beantworten: Wer hat die Informationen verbreitet? Woher stammen die Informationen? Wie plausibel sind die Informationen? Welchen Zweck verfolgt der Absender der Informationen? Werden die Informationen durch unabhängige Quellen bestätigt? Es entsteht der Eindruck, dass es nicht mehr nur eine Wahrheit gibt, sondern multiple Wahrheiten und, dass uns zunehmend die Werkzeuge dazu fehlen, wahr von unwahr zu unterscheiden.

Vor diesem gesellschaftspolitischen Hintergrund liefert das Buch „Calling Bullshit“ der beiden Professoren Bergstrom und West einen wichtigen Beitrag zur Aufklärung und Vermeidung von
Bullshit (Schwachsinn, Blödsinn, Quatsch). Schon vor Jahren haben die beiden Hochschullehrer einen Studentenkurs aufgebaut, der sich mit der Erkennung und Bekämpfung von Desinformationen beschäftigt. Die beiden Autoren haben diesen Kurs nun einer breiten Allgemeinheit zugänglich gemacht und in ein sehr unterhaltsames und informatives Buch gegossen.

In insgesamt 11 Kapiteln analysieren die Autoren alle Facetten der Desinformation und versorgen uns gleichzeitig mit einfachen und nützlichen intellektuellen Werkzeugen, Bullshit zu erkennen und zu bekämpfen. Besonders lobenswert an diesem Buch ist, dass sich die Autoren sehr viel Mühe gegeben haben, die verschiedenen Aspekte von Bullshit mit anschaulichen Beispielen aus den unterschiedlichsten Lebensbereichen zu illustrieren. Es ist aber ein Irrtum anzunehmen, dass Bullshit nur in social media und der Politik vorkommt. Nein, auch in der Wissenschaft gibt es inn. Einer der wesentlichen Gründe für das Vorhandensein von Bullshit in der Wissenschaft sind quantitative Bewertungssysteme, die aus persönlichem Ehrgeiz dazu verleiten, diese zu manipulieren und damit letztlich unbrauchbar zu machen. Das Preis-LeistungsVerhältnis des Buchs stimmt und es ist eine wirklich wichtige und unterhaltsame Wochenendlektüre für jeden Wissenschaftler.

Roland Seifert, Medizinische Hochschule Hannover, seifert.roland@mh-hannover.de Diese Rezension erscheint Open Access. ${ }^{*}$
Funding note: Open Access funding enabled and organized by Projekt DEAL. Open Access: Dieser Artikel wird unter der Creative Commons Namensnennung 4.0 International Lizenz veröffentlicht, welche die Nutzung, Vervielfältigung, Bearbeitung, Verbreitung und Wiedergabe in jeglichem Medium und Format erlaubt, sofern Sie den/die ursprünglichen Autor(en) und die Quelle ordnungsgemäß nennen, einen Link zur Creative Commons Lizenz beifügen und angeben, ob Änderungen vorgenommen wurden. Die in diesem Artikel enthaltenen Bilder und sonstiges Drittmaterial unterliegen ebenfalls der genannten Creative Commons Lizenz, sofern sich aus der Abbildungslegende nichts anderes ergibt. Sofern das betreffende Material nicht unter der genannten Creative Commons Lizenz steht und die betreffende Handlung nicht nach gesetzlichen Vorschriften erlaubt ist, ist für die oben aufgeführten Weiterverwendungen des Materials die Einwilligung des jeweiligen Rechteinhabers einzuholen. Weitere Details zur Lizenz entnehmen Sie bitte der Lizenzinformation auf

http://creativecommons.org/licenses/ by $/ 4.0 /$ deed.de. 